\title{
Burnout among caregivers in the era of the COVID-19 pandemic: Insights and challenges
}

\author{
Julie Franc-Guimond, MD; Valérie Hogues, MD \\ Division of Pediatric Urology, CHU Sainte-Justine; Department of Surgery, University of Montreal, Montreal, QC, Canada; CHUM, University of Montreal, Montreal, QC, Canada
}

Cite as: Franc-Guimond J, Hogues V. Burnout among caregivers in the era of the COVID-19 pandemic: Insights and challenges. Can Urol Assoc J 2021;15 (6Suppl1):S16-9. http://dx.doi.org/10.5489/ cuaj.7224

\section{Abstract}

The arrival of COVID-19 modified the way we live, care, teach, and self-care tremendously. Indeed, our personal and professional lives were much affected, and despite the fact that Canadian healthcare workers (HCW) and systems had some time to prepare in comparison to other countries, we faced a scenario that resembled no other. Adding the negative effects of a never-before-seen pandemic to the already well-known risk of burnout among HCW could surely increase the likelihood of colleagues, as well as ourselves, being affected. We hereby reviewed the numerous factors that could increase the risk of burnout amid this ongoing pandemic.

\section{The rise of COVID-19 or how our lives have changed}

The last months have been far from traditional and filled with several challenges. The rise of the COVID-19 epidemic, soon to become a pandemic, gave rise to a new, never-seen battlefield, with battle buddies at risk of getting sick or, even worse, dying. Indeed, some did.

Despite uncertain times, spirits were initially lifted by a massive tsunami of rainbows drawn or painted here and there, while people from the food industry made sure to keep the health workers' stomachs filled. Little did we know that some restaurants would later have to close, as many owners struggled to survive the significant financial crisis that came with these unprecedented times. Many others from various industries have suffered the same outcome. As people working in the medical field, we did not fear losing our jobs as much, but we certainly witnessed others who did, whether it be our spouses, family members, friends, neighbours, or patients.

Although we initially thought that the COVID-19 pandemic could compare to the 2009 influenza A H1N1 pandemic or to the first pandemic threat of a deadly coronavirus that emerged in late 2002 and caused an outbreak of severe acute respiratory syndrome (SARS), it certainly turned out to be much worse. ${ }^{1}$
COVID-19 has had a tremendous impact on everyone, including healthcare workers (HCW), and although physicians are not often perceived as vulnerable individuals, the arrival of this pandemic posed unique challenges even to the most experienced carers. ${ }^{2}$

\section{COVID-19 and concerns for an increased risk of burnout}

B-U-R-N-O-U-T within the medical world has been a trendy subject addressed, discussed, and written about more than ever in the past few years. Adding the negative effects of a never-before-seen pandemic to the already well-known risk of burnout among HCW could increase the likelihood of colleagues, as well as ourselves, being affected. Even though we have yet to objectively register the long-term effects of the current crisis, we are already very much aware of the deleterious consequences on the medical workers and the healthcare system.

Significant stressors brought on by the pandemic posed many new challenges to HCWs on both professional and personal levels. Beyond the risk of infection, social isolation, and economic concerns that affected society at large, HCWs had to face new demands at work that often extended beyond the traditional responsibilities ${ }^{3}$ - caring for patients virtually, which many of us did, and navigating the frontlines caring for COVID-19-positive patients. Fortunately, groups of people already aware of the risks of burnout among healthcare professionals were quick to alert those who wanted to listen that along with the COVID-19 pandemic came an increased risk of exhaustion. ${ }^{4}$ Influencers sought to increase resilience and limit burnout, as well as post-traumatic stress disorder. Despite massive intuitive social awareness that the pandemic would put a strain on the healthcare systems and workers, it became obvious that we as physicians needed to take matters into our own hands early on, in most instances due to a lack of existing support. ${ }^{5}$ Fortunately, assistance eventually became available in most settings if it was wanted/needed. ${ }^{2}$

The short-term effects the pandemic has had on the physicians and other HCWs are currently being evaluated. Results obtained from preliminary studies generally showed that the introduction of COVID-19 has increased prevailing challen- 
ges that physicians face, such as increasing workload, which is directly correlated with increased burnout. However, exposure to COVID-19 does not unavoidably translate into increased burnout; ${ }^{6}$ still, concerns remain regarding the long-term effects of COVID-19 on medical workers.

It is clear the pandemic brought significant physical and emotional burden to HCWs who had to work in the worse settings, as observed in the countries affected most by the initial wave, such as Italy and Spain. Underprepared healthcare systems faced countless challenges, an event comparable to what happens when a natural disaster occurs. ${ }^{7}$ A thorough assessment of the literature looking at wellness revealed consistent reports of stress, disturbed sleep, and depressive symptoms among medical staff because of COVID-19. The review also identified significant anxiety regarding patient care, in addition to the possibility of staffs infecting their families. Indeed, the risk of being exposed to COVID-19 at work translated in major impacts on physicians' home lives. ${ }^{8}$

Another important review highlighted the need for appropriate personal protective equipment, which remains of utmost importance to help physicians feel physically safe. ${ }^{9}$ These findings were echoed by many other publications, which also proposed appropriate psychological measures to help build resilience and offer preventative measures to limit the impact on the mental health of healthcare professionals. ${ }^{10}$ Interestingly, in some instances, being sidelined increased the risk of burnout because HCWs felt helpless.

Frontline workers were also found to have a stronger feeling of personal accomplishment as they confronted the current outcomes of their care for individuals infected by COVID-19. ${ }^{11}$ Although some professionals may have found ways to emotionally survive amid the pandemic, others are struggling. Risk of burnout, depression, substance abuse, and suicide among some HCWs appear heightened on a short- and on a long-term basis. Moreover, the effects of massive exposure to death, along with events that disturb personal principles and add moral injuries should not be undervalued, as the potential for depression and post-traumatic stress disorder is real, especially for those who saw the worst of the pandemic. ${ }^{12,13}$

\section{COVID-19 pandemic in Canada}

Despite data outlining diminished physicians' well-being due to the pandemic, most local reports have been somewhat reassuring. This could be explained by the fact that Canada had some time to prepare and that the initial Canadian wave was not as significant as in other countries. An interesting Canadian study ${ }^{14}$ describe the psychological effects of working as a Canadian emergency physician dur- ing the first weeks of the COVID-19 pandemic. Emergency physicians ( $\mathrm{n}=468$ working in 143 Canadian hospitals) completed a weekly survey. The primary outcome was physician burnout as measured by the emotional exhaustion and depersonalization items from the Maslach Burnout Inventory (MBI). Information regarding work patterns, aerosolizing procedures, testing, and diagnosis of COVID-19 were gathered. Burnout levels did not significantly change over time (emotional exhaustion $p=0.632$, depersonalization $\mathrm{p}=0.155)$. Three of 468 respondents were diagnosed with COVID-19. Being tested for COVID-19 and the number of shifts worked were linked to increased emotional exhaustion. Having been tested for COVID-19 was also associated with increased depersonalization. Personal safety, academic and educational work, personal protective equipment, the workforce, patient volumes, work patterns, and work environment had an impact on physician wellbeing. A new fiscal reality and distinct negative and positive experiences affected participants' psychological health. So, even though burnout levels remained stable, COVID-19 did impact the work environment and personal perceptions and fears, hence affecting physicians' well-being.

Although Canada fared well compared to other countries, no one can take away the fact that the first wave was particularly difficult in some provinces; for areas less affected, awaiting the arrival of "their" first wave was not necessarily any easier. Stress, anxiety, difficult settings, and increased workload have been part of our new professional life for over a year now, and although the number of infections with COVID-19 have been relatively low in Canada, too many have died and many of us know someone who did.

Beyond our fears and losses, we had to continue to do what we know best: care, give back, teach.

\section{Surgeons' lives during the COVID-19 pandemic}

As surgeons, even if we were not initially expected to care for COVID-19 patients in dedicated units, we at least had to care for those who presented with a surgical pathology, which in some cases, meant that we had to operate looking like astronauts! Some surgeons also got redeployed and assumed new roles to address increased workload and cover for lack of available personnel. This certainly meant that new leadership skills had to be exploited. Not working directly on the frontlines did not translate into being spared. Indeed, all practices had to change.

Surgeons saw challenges such as slowdown and shutdown of non-urgent surgical procedures ${ }^{15}$ and rapidly recognized the uncertainties linked to performing elective surgical procedures beyond the first wave of the pandemic. 
For some, the initial decreased workload forced upon them allowed for introspective opportunities and a chance to revise priorities. Hence, some surgeons may have identified facets of their professional life they wished to change. ${ }^{16}$

\section{Virfual ways to care, teach, perform administrative tasks, and assist each other}

Sustaining primary care during the pandemic has certainly been a challenge for all, Canadian urologists included. Virtual care has become an alternative to the traditional ways of caring for our patients, but staying in touch with them has not necessarily been easy. Early in the process, a European study revealed that many urological patients had risk factors for a poor outcome from COVID-19 and most preferred telemedicine encounters at that time. ${ }^{17}$ However, we have yet to evaluate the results for continued care maintained at a distance. A French study published early on, outlined that the current situation is leading to major disturbances in patient care in many fields of urology. ${ }^{18}$ However, an Italian article published afterwards painted a more positive picture of the virtual care given to patients presenting with a wide range of urological issues. ${ }^{19}$

There have also been concerns regarding additional risks of clinician burnout due to screen fatigue, possible loss of crucial information, and the struggle to develop and maintain - at a distance - a strong patient-clinician relationship, especially if sensitive discussions need to occur. ${ }^{20,21}$ Despite likely pitfalls linked to virtual care, new ways to ensure remote healthcare could remain an option in the future and should be explored further. ${ }^{22}$

Another challenge has been to maintain support to the trainees. Quarantines, reassignments, and suspension of regularly scheduled meetings meant we had to adjust rapidly. Hence, urologists used web-based platforms to teach virtually, which at times, may have been stressful. And though the long-term impact of the pandemic on urology training is undetermined, some good may come of it. ${ }^{23,24}$ Despite required adjustments, it appears that virtual platforms initially used to care, teach, and even perform administrative tasks ${ }^{25}$ have at least reduced the helplessness experienced by physicians during these extraordinary circumstances. ${ }^{26}$

With time, various Canadian organizations and others worldwide managed to offer support to HCWs in various aspects of their life. In fact, there is now a variety of available assistance that includes technical, as well as emotional support.

\section{Moving forward}

Surely, wellness and self-care must be considered important factors for HCWs themselves, as well as for the future of all healthcare delivery systems. In fact, the COVID-19 pandemic unquestionably reiterated the need to increase resilience and decrease mental illnesses for those working in the medical field. ${ }^{27}$ More recently, authors using a validated COVID-19 burnout scale ${ }^{28}$ showed that feeling well-protected, rested, and hopeful could limit the risk of burnout specifically associated with COVID-19.

For the time being, we must remember that we are simply human because navigating urology's new normal simply is not. ${ }^{29}$

\section{References}

1. Petersen E, Koopmans M, Go U, et al. Comparing SARS-CoV-2 with SARS-CoV and influenza pandemics. Lancet Infect Dis 2020;20:e238-44. htrps://doi.org/10.1016/S1473-3099(20)30484-9

2. Dewey C, Hingle S, Goelz E, et al. Supporting clinicians during the COVID-19 pandemic. Ann Intern Med 2020;172:752-3. https://doi.org/10.7326/M20-1033

3. Bradley M, Chahar P. Burnout of healthcare providers during COVID-19. Cleve Clin J Med 2020. Epub ahead of print. https://doi.org/10.3949/ccim.87a.ccc051

4. Shah K, Chaudhari G, Kamrai D, et al. How essential is to focus on physician's health and burnout in coronavirus (COVID-19) pandemic? Cureus 2020;12:e7538. https://doi.org/10.7759/cureus.7538

5. Simons G, Baldwin DS. Covid-19: Doctors must take control of their well-being. BMJ 2020;369. https://doi.org/10.1136/bmi.m1725

6. Amanullah S, Ramesh Shankar R. The Impact of COVID-19 on physician burnout globally: A review. Healthcare (Basel) 2020;8:421. https://doi.org/10.3390/healthcare8040421

7. Restauri N, Sheridan AD. Burnout and posttraumatic stress disorder in the coronavirus disease 2019 (COVID-19) pandemic: Intersection, impact, and interventions. J Am Coll Radiol 2020;17:921-6. https://doi.org/10.1016/i.jacr.2020.05.021

8. Rodriguez RM, Medak AJ, Baumann BM, et al. Academic emergency medicine physicians' anxiety levels, stressors, and potential stress mitigation measures during the acceleration phase of the COVID-19 pandemic. Acad Emerg Med 2020;27:700-7. https://doi.org/10.1111/acem.14065

9. Shreffler J, Petrey J, Huecker M. The Impact of COVID-19 on healthcare worker wellness: A scoping review. West J Emerg Med 2020;21:1059-66. https://doi.org/10.5811/westiem.2020.7.48684

10. Raudenská J, Steinerová V, Jav rková A, et al. Occupational burnout syndrome and post-traumatic stress among healthcare professionals during the novel coronavirus disease 2019 (COVID-19) pandemic. Best Pract Res Clin Anaesthesiol 2020;34:553-60. https://doi.org/10.1016/i.bpa.2020.07.008

11. Dinibutun SR. Factors associated with burnout among physicians: An evaluation during a period of COVID-19 pandemic. J Healthc Leadersh 2020;12:85-94. https://doi.org/10.2147/JHL.S270440

12. Kingston AM. Break the silence: Physician suicide in the time of COVID-19. Mo Med 2020;117:426-9. https://doi.org/10.1038/s41477-020-0682-7

13. Reger MA, Piccirillo ML, Buchman-Schmitt JM. COVID-19, mental health, and suicide risk among healthcare workers: Looking geyond the crisis. J Clin Psychiatry 2020;81:20com13381. https://doi.org/10.4088/JCP.20coml3381

14. de Wit K, Mercuri M, Wallner C, et al; Network of Canadian Emergency Researchers. Canadian emergency physician psychological distress and burnout during the first 10 weeks of COVID-19: A mixed-methods study. J Am Coll Emerg Physicians Open 2020;1:1030-8. https://doi.org/10.1002/emp2.12225

15. Ferreira R, McGrath M, Wang Y, et al. How to prioritize urological surgeries during epidemics: Lessons learned from the Toronto SARS outbreak in 2003. Can Urol Assoc J 2020;14:E159-60. https://doi. org/10.5489/cuai.6551

16. Romanelli J, Gee D, Mellinger JD, et al; SAGES Reimagining the Practice of Surgery Task Force. The COVID-19 reset: Lessons from the pandemic on burnout and the practice of surgery. Surg Endosc 2020;34:5201-7. htrps://doi.org/10.1007/s00464-020-08072-8 
17. Boehm K, Ziewers S, Brandt MP, et al. Telemedicine online visits in urology during the COVID19 pandemic — potential, risk factors, and patients' perspective. Eur Urol 2020;78:16-20. https://doi.org/10.1016/i.eururo.2020.04.055

18. Phé $V$, Karsenty $G$, Robert $G$, et al. Widespread postponement of functional urology cases during the COVID-19 pandemic: Rationale, potential piffalls, and future consequences. Eur Urol 2020;78:4-5. https://doi.org/10.1016/i.eururo.2020.04.031

19. Novara G, Checcucci E, Crestani A, et al; Research Urology Network (RUN). Telehealth in urology: A systematic review of the literature. How much can telemedicine be useful during and after the COVID-19 pandemic? Eur Urol 2020;78:786-811. https://doi.org/10.1016/i.eururo.2020.06.025

20. Shachak A, Alkureishi MA. Virtual care: A 'Zoombie' apocalypse? J Am Med Inform Assoc 2020;27:18135. https://doi.org/10.1093/jamia/ocaal85

21. Patel HV, Srivastava A, Tabakin AL, et al. The patient-urologist relationship in the COVID-19 era and beyond. Can Urol Assoc J 2020;14:E271-3. https://doi.org/10.5489/cuaj.6688

22. Sosnowski R, Kamecki H, Joniau $S$, et al. Introduction of telemedicine during the COVID19 pandemic: A challenge for now, an opportunity for the future. Eur Urol 2020;78:820-1. https://doi.org/10.1016/i.eururo.2020.07.007

23. Smigelski M, Movassaghi M, Small A. Urology virtual education programs during the COVID-19 pandemic. Curr Urol Rep 2020;21:50. https://doi.org/10.1007/s1 1934-020-01004-y

24. Gabara A, Visram K, Leveridge M. Early adaptation of urology residency educational programs during COVID-19 clinical and gathering restrictions. Can Urol Assoc J 2020;14:235-6. https://doi.org/10.5489/cuaj.6746
25. Sullivan EE, Phillips RS. Sustaining primary care teams in the midst of a pandemic. Isr J Health Policy Res 2020;9:77. https://doi.org/10.1186/s13584-020-00434-w

26. Moazzami B, Razavi-Khorasani N, Dooghaie Moghadam A, et al. COVID-19 and telemedicine: Immediate action required for maintaining healthcare providers well-being. J Clin Virol 2020;126:104345. https://doi.org/10.1016/i.jcv.2020.104345

27. Yildrim $M$, Arslan G, Özaslan A. Perceived risk and mental health problems among healthcare professionals during COVID-19 pandemic: Exploring the mediating effects of resilience and coronavirus fear. Int J Ment Health Addict 2020:1-11. https://doi.org/10.31234/osf.io/84xiu

28. Yildrrm M, Solmaz F. COVID-19 burnout, COVID-19 stress. and resilience: Initial psychometric properties of COVID-19 burnout scale. Death Stud 2020 Sep 11:1-9. https://doi.org/10.1080/07481 187.2020.1818885

29. MacDonald L, Cox A, Jarvi K, et al. Navigating urology's new normal and mitigating the effects of a second wave of COVID-19. Can Urol Assoc J 2020; 14:E543-8. htrps://doi.org/10.5489/cuaj.6976

Correspondence: Dr. Julie Franc-Guimond, Division of Pediatric Urology, CHU Sainte-Justine; Department of Surgery, University of Montreal, Montreal, QC, Canada; ffrancguimond@yahoo.ca 